THÉORIE DES NOMBRES

BESANÇON

The Hasse invariant of the trace form of a central simple algebra 


\title{
THE HASSE INVARIANT OF THE TRACE FORM OF A CENTRAL SIMPLE ALGEBRA
}

\author{
David W. LEWIS AND Jorge F. Morales
}

\begin{abstract}
A.ssruct. This paper shows that the Hasse invarient of the trace form of a central simple algebra $A$ is related by a simple formula to the class of $A$ in the Brauer group.
\end{abstract}

\section{INTRODUCTION}

Trace forms of central simple algebras have been studied recently by Rowen [4] and Formanek [1] in connection with Brauer factor sets. Also, in [3], the determinant and signatures of these trace forms were calculated in general. In a few special cases the Clifford invariant was calculated but a calculation in general seemed inaccessible. However, by using algebraic groups and Galois cohomology, we are able in this paper to determine in general the Hasse invariant of the trace form of a central simple algebra. The Hasse invariant happens to coincide with the Clifford invariant for these trace forms. Our viewpoint also yields a simple, alternative way of calculating the determinant of a trace form.

Our formula (2) is the analogue for central simple algebras of Serre's formula [7, Théorème 1] for étale algebras. In Serre's proof, the two-fold cover $\widetilde{\mathfrak{S}}_{n}$ of the symmetric group $\mathfrak{S}_{n}$ plays a crucial role. The group $\widetilde{\mathfrak{S}}_{n}$ is characterized by the fact that the natural orthogonal representation $\mathfrak{S}_{n} \rightarrow O_{n}$ can be lifted to a "spinorial" representation $\widetilde{\mathfrak{S}}_{n} \rightarrow \widetilde{O}_{n}$, where $\widetilde{O}_{n}$ is a two-fold cover of $O_{n}$ containing the spinor group $\operatorname{Spin}_{n}$ as a subgroup of index 2. In the case of central simple algebras, we use the analogous fact that the adjoint representation $P G L_{n} \rightarrow S O_{n^{2}}$ can be lifted to a spinorial representation $S L_{n} \rightarrow S p i n_{n} 2$.

Addendum. After writing this paper, Jean-Pierre Tignol pointed out to us that Theorem 1 had been announced by Saltman at a 1987 Berkeley mini-conference on Division Algebras and Quadratic Forms. Saltman's proof uses generic splitting fields and seems to be completely different to ours. To the best of our knowledge, Saltman did not publish his proof.

It was also pointed out to us that Serre had proved the same result in his 1990-1991 lectures at the College de France. The undetailed outline that Serre provides in [8] suggests that our proof is on the same lines as his.

We must therefore acknowledge that the main result of this paper is not new. We would like to state unambiguously that we do not have any priority claims. Several colleagues have asked us to publish this paper in despite of the facts mentioned above, as a service to the mathematical community. Jean-Pierre Tignol is providing a different proof in the same issue of this journal.

1991 Mathematics Subject Classification. Primary 11E04; Secondary 12G05, 13 A20.

The second author was partially supported by National Science Foundation grant DMS-9205129. 


\section{TRACE FORMS OF CENTRAL SIMPLE ALGEBRAS}

The following notation will be in force throughout this paper:

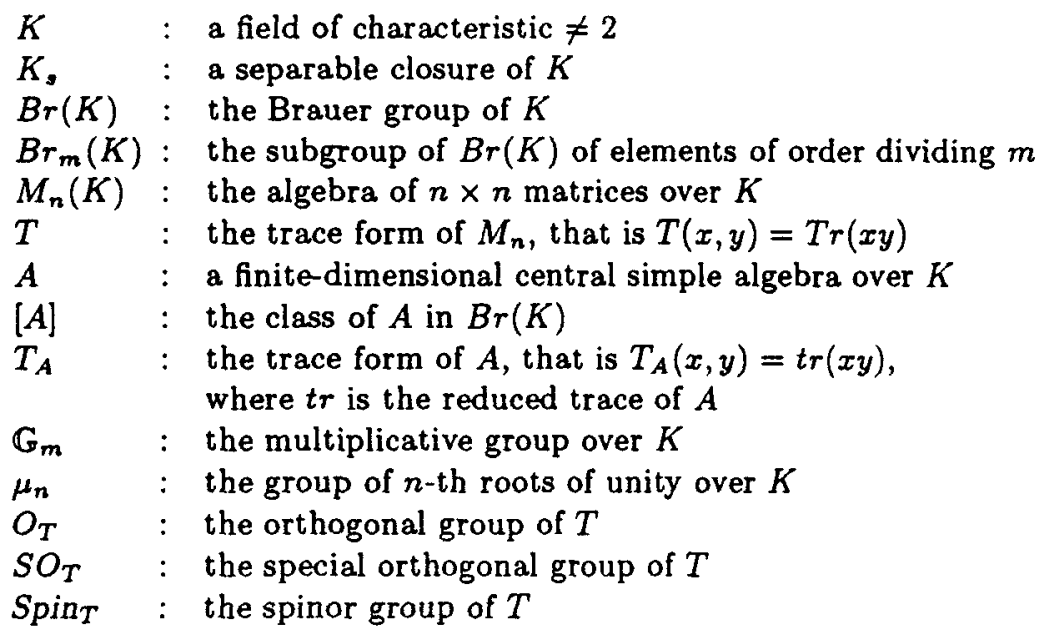

Let $B$ be a nondegenerate symmetric bilinear form over $K$ and let $\left.<a_{1}, a_{2}, \ldots, a_{m}\right\rangle$ be a diagonalization of $B$. Recall that the determinant of $B$ is defined by

$$
d(B)=\prod_{i=1}^{m}\left(a_{i}\right) \in K^{\times} / K^{\times 2}=H^{1}\left(K, \mu_{2}\right),
$$

and that the Hasse invariant of $B$ is

$$
h(B)=\bigotimes_{1 \leq i<j \leq m}\left(\frac{a_{i}, a_{j}}{K}\right) \in B r_{2}(K)=H^{2}\left(K, \mu_{2}\right) .
$$

It is a standard fact that the above definitions do not depend upon the particular diagonalization chosen for $B$ (see, for instance, [5, Chap. 1, 3.17 and Chap 2, 12.5]).

Our goal is to compute $h\left(T_{A}\right)$ and, incidentally, $d\left(T_{A}\right)$ for the trace form $T_{A}$ of a central simple algebra $A$ over $K$.

If $A$ is the split algebra $M_{n}(K)$, an elementary computation using the standard basis $\left\{e_{i j}\right\}$ shows

$$
d(T)=(-1)^{n(n-1) / 2} \text { and } h(T)=\left(\frac{-1,-1}{K}\right)^{(n-2)(n-1) n(n+1) / 8} .
$$

It is also easy to see, using the formulae in [5, page 81], that for an arbitrary $A$, the Hasse invariant $h\left(T_{A}\right)$ coincides with the Clifford invariant $C\left(T_{A}\right)$.

Suppose now that $A$ has dimension $n^{2}$ over $K$ and let $L \subset A$ be a maximal commutative subfield. It is well known that $L$ has degree $n$ over $K$ and that it is a splitting field for $A$. Thus, if $n$ is odd then, by Springer's Theorem (see [5, Chap. 2, 5.4]), $T_{A}$ is isometric to the trace form $T$ of $M_{n}(K)$. Hence the interesting case is when $n$ is even.

Here is our main result: 
Theorem 1. Let $A$ be a central simple algebra over $K$ of even dimension $n^{2}$. Then

$$
h\left(T_{A}\right)=[A]^{n / 2}\left(\frac{-1,-1}{K}\right)^{(n-2) n / 8}
$$

(recall that $[A]$ denotes the class of $A$ in the Brauer group $B r(K)$ ).

Before proving this theorem we shall recall a few elementary facts about Galois cohomology and the theory of descent. It is well-known (see, for instance, [6, Chapter X, §5]) that the cohomology set $H^{1}\left(K, A u t\left(M_{n}\right)\right)$ classifies the central simple algebras of dimension $n^{2}$ over $K$. The short exact sequence

$$
1 \rightarrow \mathbb{G}_{m} \rightarrow G L_{n} \stackrel{\operatorname{Inn}}{\longrightarrow} \operatorname{Aut}\left(M_{n}\right) \rightarrow 1,
$$

where $\operatorname{Inn}(a)$ is the inner automorphism $\operatorname{In} n(a)(x)=a x a^{-1}$, induces an injective map in cohomology

$$
H^{1}\left(K, \operatorname{Aut}\left(M_{n}\right)\right) \stackrel{\theta}{\rightarrow} H^{2}\left(K, \mathbb{G}_{m}\right)=B r(K) .
$$

The map $\partial$ takes every simple central algebra over $K$ of dimension $n^{2}$ to its class in the Brauer group $B r(K)$.

The cohomology set $H^{1}\left(K, O_{T}\right)$ classifies the symmetric bilinear forms $B$ over $K$ that are isometric to $T$ over the separable closure $K_{s}$. The class of $T$ corresponds to the "neutral element" in $H^{1}\left(K, O_{T}\right)$. We have two short exact sequences related to the orthogonal group

$$
1 \rightarrow S O_{T} \rightarrow O_{T} \stackrel{\text { det }}{\longrightarrow} \mu_{2} \rightarrow 1,
$$

and

$$
1 \rightarrow \mu_{2} \rightarrow \operatorname{Spin}_{T} \rightarrow S O_{T} \rightarrow 1
$$

From these sequences one obtains maps

$$
\operatorname{det}_{*}: H^{1}\left(K, O_{T}\right) \rightarrow H^{1}\left(K, \mu_{2}\right)=K^{\times} / K^{\times 2},
$$

and

$$
\partial: H^{1}\left(K, S O_{T}\right) \rightarrow H^{2}\left(K, \mu_{2}\right)=B r_{2}(K) .
$$

These maps are related to the determinant $d$ and the Hasse invariant $h$ by the following formulae:

$$
d(B)=\operatorname{det}_{*}(B) d(T),
$$

and

$$
h(B)=\partial(B) h(T) .
$$

(Identity (3) is obvious from the definition and (4) can be easily deduced from [9, Theorem 4.4 and (4.7)]).

The group $\operatorname{Aut}\left(M_{n}\right) \simeq P G L_{n}$ acts by isometries on $\left(M_{n}, T\right)$, that is $\operatorname{Aut}\left(M_{n}\right)$ is a subgroup of $O_{T}$. Let $\iota: \operatorname{Aut}\left(M_{n}\right) \rightarrow O_{T}$ be the natural inclusion and let

$$
\iota_{*}: H^{1}\left(K, A u t\left(M_{n}\right)\right) \rightarrow H^{1}\left(K, O_{T}\right)
$$

be the map induced by $\iota$ in cohomology. 
Proposition 2. Let $A$ be a central simple algebra over $K$ and let $T_{A}$ be its trace form. Then $\iota_{*}(A)=T_{A}$.

Proof. Let $\phi: M_{n}\left(K_{s}\right) \rightarrow A \otimes K$, be an algebra isomorphism. The class of $A$ in the cohomology set $H^{1}\left(K, A u t\left(M_{n}\right)\right)$ is represented by the 1 -cocycle $c_{A}(\omega)=\phi^{-1} \circ{ }^{\omega} \phi\left(\omega \in G a l\left(K_{s} / K\right)\right)$. Since $\phi$ is an algebra isomorphism, it must preserve the trace forms, hence $\iota \circ c_{A}$ represents the class of $T_{A}$ in $H^{1}\left(K, O_{T}\right)$.

Proposition 2 can be used to give another proof of Theorem 1.3 of [3]:

Corollary 3. $d\left(T_{A}\right)=d(T)=(-1)^{n(n-1) / 2}$.

Proof. Since $A u t\left(M_{n}\right)$ is a connected algebraic group, it is contained in $S O_{T}$, i.e. the composite homomorphism

$$
\operatorname{Aut}\left(M_{n}\right) \stackrel{\iota}{\rightarrow} O_{T} \stackrel{\text { det }}{\longrightarrow} \mu_{2}
$$

is trivial. Thus $\operatorname{det}_{*}\left(T_{A}\right)=\operatorname{det}_{*} \iota_{*}(A)=1$. We conclude from (3) that $d\left(T_{A}\right)=d(T)$. By (1), $d(T)=(-1)^{n(n-1) / 2}$.

Since $S p i n_{T}$ is the universal cover of $S O_{T}$ and $S L_{n}$ is simply connected, there exists a homomorphism $\tau: S L_{n} \rightarrow S p i n_{T}$ such that the diagram

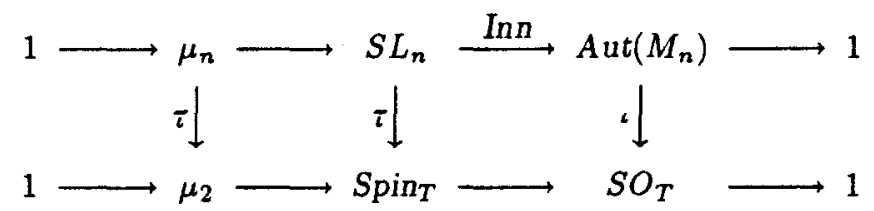

commutes.

Lemma 4. For $n$ even, the induced map $\tau: \mu_{n} \rightarrow \mu_{2}$ is non-trivial

Proof. It is sufficient to show that the map $\tilde{\imath}: \mu_{n} \rightarrow \mu_{2}$ is non-trivial over some extension of $K$. Replacing $K$ if necessary by the rational function field $K(t)$ we may assume that $K$ is not quadratically closed. Consider the following portion of the diagram arising from the cohomology exact sequences

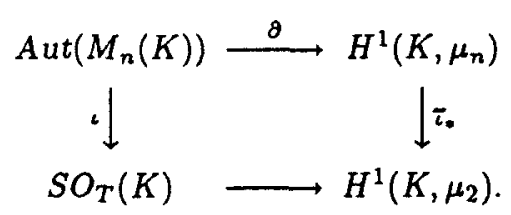

The map $S O_{T}(K) \rightarrow H^{1}\left(K, \mu_{2}\right)$ is known to be the spinor norm (see [2, page 133]). To prove the lemma it suffices to show that $\tau_{*}$ is non-trivial. By virtue of the diagram above, it is enough to produce a matrix $a \in G L_{n}(K)$ such that the spinor norm of $\operatorname{Inn}(a)$ is a non-square in $K$.

Let $a=\operatorname{Diag}(d, 1,1, \ldots, 1)$, where $d$ is in $K^{\times} \backslash K^{\times 2}$ (recall that we are assuming that $K$ is not quadratically closed). Let $x=\left(x_{i j}\right)$ be an element of $M_{n}(K)$. We have, by direct computation,

$$
\operatorname{Inn}(a)(x)=\left(\begin{array}{cccc}
x_{11} & d x_{12} & \ldots & d x_{1 n} \\
d^{-1} x_{21} & x_{22} & \ldots & x_{2 n} \\
\ldots & \ldots & \ldots & \ldots \\
d^{-1} x_{n 1} & x_{n 2} & \ldots & x_{n n}
\end{array}\right)
$$


Recall that for an an anisotropic vector $v \in M_{n}(K)$, the reflection $\tau_{v}$ with respect to the hyperplane orthogonal to $v$ is given by

$$
\tau_{v}(w)=w-2 \frac{T(v, w)}{T(v, v)} v
$$

Let $\left\{e_{i j}\right\}$ be the standard basis of $M_{n}(K)$ and set

$$
\begin{aligned}
v_{i} & =e_{i 1}-d e_{1 i} \\
w_{i} & =e_{i 1}-e_{1 i}
\end{aligned}
$$

for $i=2,3, \ldots, n$. By direct computation we have

$$
r_{v_{i}} \tau_{w_{i}}(x)=\left(\begin{array}{ccccc}
x_{11} & \ldots & d x_{1 i} & \ldots & x_{1 n} \\
\ldots & \ldots & \ldots & \ldots & \ldots \\
d^{-1} x_{i 1} & \ldots & x_{i i} & \ldots & x_{i n} \\
\ldots & \ldots & \ldots & \ldots & \ldots \\
x_{n 1} & \ldots & x_{n i} & \ldots & x_{n n}
\end{array}\right)
$$

Hence

$$
\operatorname{Inn}(a)=\tau_{v_{2}} \tau_{w_{3}} \tau_{v_{3}} \tau_{w_{3}} \ldots \tau_{v_{n}} \tau_{w_{n}}
$$

This identity allows us to compute the spinor norm of $\operatorname{Inn}(a)$ :

$$
\text { Spinor norm of } \begin{aligned}
\operatorname{Inn}(a) & \equiv \prod_{i=2}^{n} T\left(v_{i}, v_{i}\right) T\left(w_{i}, w_{i}\right)\left(\bmod K^{\times 2}\right) \\
& \equiv \prod_{i=2}^{n}(-2 d)^{n-1}(-2)^{n-1}\left(\bmod K^{\times 2}\right) \\
& \equiv d\left(\bmod K^{\times 2}\right)
\end{aligned}
$$

Since $d$ was chosen to be a non-square in $K$, the above computation finishes the proof of the lemma.

We are now ready to prove Theorem 1 .

Proof of Theorem 1. By Lemma 4, the map $\tau: \mu_{n} \rightarrow \mu_{2}$ is given by $\tau(\xi)=\xi^{n / 2}$. Hence the map $\tau_{*}: H^{2}\left(K, \mu_{n}\right) \rightarrow H^{2}\left(K, \mu_{2}\right)$ induced in cohomology is given by $\tau_{*}[A]=[A]^{n / 2}$ (note that the natural inclusion $\mu_{2} \rightarrow \mu_{n}$ induces an injection $H^{2}\left(K, \mu_{2}\right) \rightarrow H^{2}\left(K, \mu_{n}\right)$ ). From (5) we obtain a commutative diagram in cohomology

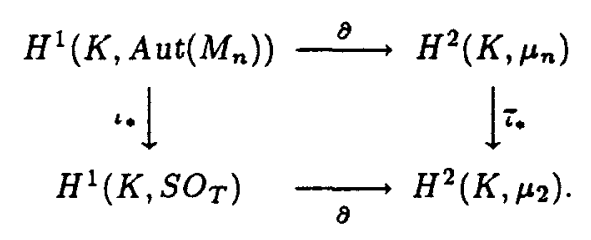

By Proposition 2 and the diagram above we have $\partial\left(T_{A}\right)=\tau_{*} \partial(A)=[A]^{n / 2}$. We conclude by using identities (4) and (1). 


\section{REFERENCES}

1. Formanek, E., Some remarks about the reduced trace, Israel Math. Conf. Proc., Ring Theory 1989, Vol. 1, Weizmann Science Press of Israel, 1989, pp. 337-343.

2. Kneser, M., Galois Cohomology of Classical Groups, Tata Institute Lecture Notes, Bombay, 1969.

3. Lewis, D.W., Trace forms of central simple algebras, Math. Z. (to appear).

4. Rowen, L.H, Brauer factor sets and simple algebras, Trans A.M.S 282 (1984), 767-772.

5. Scharlsu, W., Quadratic and Hermitian Forms, Springer-Verlag, New York-Heidelberg-Berlin, 1985.

6. Serre, J.-P., Local Fields, Springer-Verlag, New York-Heidelberg-Berlin, 1979.

7. Serre, J.-P., L'invariant de Witt de la forme $\operatorname{Tr}\left(x^{2}\right)$, Comment. Math. Helvetici 59 (1984), 651-676.

8. Serre, J.-P., Résume des cours,, Collège de France (1990-91).

9. Springer, T.A., On the equivalence of quadratic forms, Indag. Math. 21 (1959), 241-253.

Uhivensty College Dublim, Departuext of Mathexatics, Dqbin 4, Irelahd

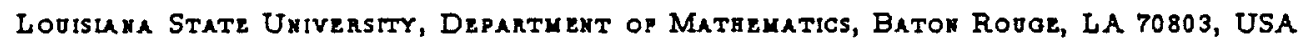

